\title{
What potential do magnetic iron oxide nanoparticles have for the treatment of rheumatoid arthritis?
}

\author{
Lin $\mathrm{Wu}^{1}$ \& Song Shen $*, 2$ (iD \\ ${ }^{1}$ Department of Pharmacy, Affiliated Hospital of Jiangsu University, Zhenjiang 212001, China \\ ${ }^{2}$ Department of Pharmaceutics, School of Pharmaceutical Science, Jiangsu University, Zhenjiang 212013, China \\ *Author for correspondence: jsdx.shensong@163.com
}

"The application of IONPs in RA therapy not only provides an effective approach for RA diagnosis and treatment but may also encourage the development of novel magnetic materials with higher performance potential."

First draft submitted: 15 February 2019; Accepted for publication: 22 February 2019; Published online: 29 March 2019

Keywords: magnetic hyperthermia therapy • magnetic iron oxide nanoparticles $\bullet$ magnetic resonance imaging • photothermal therapy $\bullet$ reactive oxygen species $\bullet$ rheumatoid arthritis $\bullet$ targeting drug delivery

Rheumatoid arthritis (RA) is a systemic autoimmune disease affecting $0.5-1.0 \%$ of the adult population worldwide [1]. It is characterized by pannus formation, synovial inflammation and hyperplasia, autoantibody production, cartilage and bone destruction. During the pathologic progression, angiogenesis contributes to the proliferation of the inflammatory synovial pannus as well as to the infiltration of the pannus by inflammatory leucocytes. However, the newly formed vessels are usually abnormal in architecture and have defective endothelial cells, making the vessels permeable to large particles. In addition, the overexpressed inflammatory mediators such as TNF- $\alpha$ in diseased tissue can also impair the integrity of the endothelial lining and increase permeability [2]. This enhanced permeability and retention effect opens a window for the application of nanoparticles in the early diagnosis and specific therapy of RA.

Magnetic iron oxide nanoparticles (IONPs) have attracted significant interest in biological applications owing to their low toxicity and good biocompatibility. They are the only metal oxide nanoparticles approved by US FDA for clinical use as magnetic resonance imaging (MRI) contrast agents [3]. They also show great biodegradability; ultrasmall IONPs $(<10 \mathrm{~nm})$ can be removed by renal clearance while large IONPs will dissolve in acidic lysosomes to form elemental iron which can be subsequently incorporated into ferritin proteins [4]. One exciting property of IONPs is their unique superparamagnetism, which enables the nanoparticles to simultaneously serve as carriers for targeted drug delivery, as MRI contrast agents for diagnosis and as transducers for hyperthermia therapy. Meanwhile, ferrous ions also participate in the Fenton reaction in the acidic environment to generate highly toxic hydroxyl radicals, offering a novel approach for RA therapy. All these functions make the IONPs a good candidate for stimuli-responsive drug release and combined therapy (e.g., chemo-hyperthermia therapy) to achieve enhanced therapeutic effects and reduced side effects. In this editorial, we will highlight the strategies and applications of IONPs in RA therapy.

\section{RA tissue targeting}

IONPs have been extensively used as carriers for targeted delivery of chemotherapeutic drugs (e.g., methotrexate) and genes (e.g., siRNA). The accumulation of IONPs in arthritic tissue can be achieved by passive and/or active targeting effect of the nanoparticles. In passive targeting, IONPs reach the RA tissue by the enhanced permeability and retention effect. Our study indicated that IONPs with a size of $220 \mathrm{~nm}$ were more efficient in RA targeting than the counterparts with sizes of 70, 110 and $350 \mathrm{~nm}$ [5]. A possible explanation for this phenomenon was the cut-off size effect of the endothelial gaps for large particles. The small particles could easily penetrate into 
the RA tissue but also readily diffuse out, resulting in relatively low accumulation in the inflamed joint. To improve their therapeutic effect, the nanoparticles should be internalized by cells at the disease site. Outcomes in cell internalization can be quite different, in that smaller IONPs are more readily uptaken by RA cells than larger ones, this is consistent with other nanoparticles [6]. Aside from size, RA targeting effect and cell uptake are influenced by surface hydrophilicity and surface charge. The hydrophilic surface protects the IONPs from being removed by reticuloendothelial system, resulting in longer circulation and greater targeting efficiency. However, the hydrophilic surface will also prevent the internalization of cells due to the weak affinity toward the cell membrane. Positively charged IONPs were usually phagocytized more efficiently because of the electrostatic interaction with the negatively charged membrane. However, they tend to be recognized by reticuloendothelial system and quickly cleared in circulation.

In active targeting, IONPs display significant advantages in that they can be magnetically guided to desired tissues using an external magnet. Furthermore, IONPs can be modified with various targeting ligands/antibodies (e.g., $\alpha_{v} \beta_{3}$ integrins, EGFRs) on the surface to enhance the specific targeting effect.

Although passive and active targeting delivery can increase the therapeutic effect, the distribution of the nanoparticles in normal tissue is inevitable. Intelligent carriers that can specifically release drugs in targeted sites should be developed as an efficient approach to enhance treatment effect. IONPs exhibit significant potential for smart drug delivery due to their ability to respond to various stimuli. Endogenous stimuli such as acidic environment can trigger the dissolution of IONPs and subsequently cause the drug release, suggesting a potential acidotic RA synovial fluids targeting strategy [7]. While exogenous stimuli, including magnetic fields and light, can trigger the burst release of drug at targeted sites by the magneto calorific effect and photothermal effects of IONPs. This smart drug delivery provides a novel approach for precise treatment, which not only prevents the premature release in blood circulation but also accelerates drug release in disease areas.

\section{IONPs for RA MRI diagnosis}

MRI is recognized as the gold standard for synovial diagnosis, presenting superior sensitivity and accuracy over other clinical signs [8]. Conventionally, gadolinium complexes (typically Gd-DTPA) are used as the standard contrast agent to differentiate synovial hyperplasia from other tissues by $T_{1}$-weighted images. However, the specificity of GdDTPA enhancement in the synovium is limited because of the indiscriminate accumulation in inflammatory tissues and normal joints. Besides, the molecular weight of Gd-DTPA is low, leading to the quick elimination and short residence time in vivo [9]. In contrast, superparamagnetic iron oxide nanoparticles (SPIONs) (defined as IONPs with diameters ranging from several nanometers to $>100 \mathrm{~nm}$ ) can penetrate into the inflamed joint efficiently and further be internalized by the inflammatory cells within the synovium, showing a specific accumulation and persistent MRI signal enhancement for arthritic joints [8]. Commercially available SPIONs (SHU555C) presented a longer enhanced MRI effect in the inflamed joint than by Gd-DTPA [9]. Generally, SPIONs are used as $T_{2}$ contrast agents. However, once the size deceased, the $T_{2}$-weighted imaging activity will be weakened together with the increase of $T_{1}$-weighted imaging activity [10]. Therefore, ultrasmall SPIONs show potential to act as a $T_{1}$ contrast agent. $T_{1}$-effect of SPIONs in vivo is normally considered to be inferior to that of gadolinium complexes. Nevertheless, a study performed by Simon et al. indicated a comparable $T_{1}$-enhancement effect of SPIONs (SHU555C) to Gd-DTPA [9]. What is more, SHU555C showed prolonged enhancement and a greater difference between arthritic and normal synovium. Hence, SPIONs present a great prospect in both $T_{1}$ - and $T_{2}$-weighted imaging for RA diagnosis.

\section{IONPs for RA hyperthermia therapy}

In the pathological progression of RA, the aggressive front of abnormal tissue, the pannus, actively participates in joint inflammation and contributes directly to local cartilage and bone destruction displaying tumor-like behaviors, such as apoptosis resistance, increased aggressiveness and invalid contact inhibition [1]. Thus, hyperthermia is expected to be useful for RA treatment. Magnetic hyperthermia therapy (MHT) is a minimally invasive approach for RA therapy. With the help of an external alternating magnetic field, IONPs can generate heat efficiently through the mechanism of Brownian-Néel relaxation and hysteresis losses. Although IONPs exhibit superior magnetic thermal induction effect to other metal particles, the conversion efficiency is not satisfied yet, which hinders the therapeutic effect and the widespread use of MHT in a clinical setting. To further improve the magnetic heating effect, IONPs can be designed to construct core-shell structured nanocomposites to increase the specific loss power [11]. In the hyperthermia therapy, temperature is expected to be controlled within the range of $42-47^{\circ} \mathrm{C}$, since 
over high temperature $\left(>48^{\circ} \mathrm{C}\right.$, thermal ablation) will cause damage to the surrounding normal tissue. However, there is still an absence of a noninvasive method for real-time monitoring $3 \mathrm{D}$ temperature distributions in the tissue. Although novel technologies, such as electrical impedance tomography, microwave radiometry, computer tomography (CT), magnetic resonance imaging (MRI) and ultrasound, have emerged in recent years, they have not yet been widely applicated in the clinic. Another challenge in RA hyperthermia therapy is the inhomogeneous temperature distribution in RA tissue. This phenomenon is caused by three factors: the unevenly distributed IONPs in inflamed joint, the attenuation of light during the irradiation and the cooling effect of blood.

IONPs are not only a good candidate for magnetic hyperthermia therapy but also for photothermal therapy. Our study indicated that carboxymethyl chitosan coated $\mathrm{Fe}_{3} \mathrm{O}_{4}$ with a diameter of $177 \mathrm{~nm}$ showed a comparable photothermal effect with hollow gold nanospheres and low cytotoxicity [12]. We also evaluated the potential of different-sized $\mathrm{Fe}_{3} \mathrm{O}_{4}$ nanoparticles for RA photothermal treatment. The 220-nm IONPs induced the highest temperature of $\sim 51.7^{\circ} \mathrm{C}$ and the most remarkable inflammatory inhibition rate in the inflamed joint. The significantly higher efficacy was attributed to the better inflamed joint targeting effect of 220-nm IONPs. Although $0-100 \mathrm{~nm}$ is recognized as the most prospective size for nanoparticles targeting, our study demonstrated that the optimized size for RA therapy was $220 \mathrm{~nm}$.

\section{IONPs induced hydroxyl radical for RA therapy}

Reactive oxygen species (ROS) are mutagenic and are persistently upregulated in RA tissue. The upregulated ROS can be attributed to the activation of phagocytic cells such as macrophages and neutrophils, which undergo an oxidative burst mediated by NADPH oxidase system to produce superoxide $\left(\mathrm{O}_{2}{ }^{-} \bullet\right)$ accompanying with the consumption of oxygen [13]. The generated superoxide will be then converted to hydrogen peroxide $\left(\mathrm{H}_{2} \mathrm{O}_{2}\right)$ and other oxidants, resulting in oxidative stress and the damage of lipids, proteins and DNA in joint tissues. In acidic environment, IONPs dissolve to release iron ions, which can catalyst hydrogen peroxide and superoxide to generate highly reactive hydroxyl radicals $(\mathrm{OH} \bullet)$ via Fenton and Haber-Weiss reaction [14]. The hydroxyl radicals are extremely toxic and can damage the hyperplasic pannus, indicating a potential in RA therapy. Similar therapeutic strategies have been applied in cancer therapy. It is worth noting that the therapeutic effect may be related with the concentration of formed hydroxyl radicals. Since studies also indicated that the iron presented in RA synovial tissue would result in poor prognosis [15]. Hence, more detailed work is needed to verify this novel strategy in RA therapy.

\section{Conclusion}

Altogether, IONPs are demonstrated as an effective nanoplatform for targeting drug delivery, stimuli-responsive drug release, MRI diagnosis, photothermal therapy and magnetic hyperthermia therapy in RA treatment. The versatility makes IONPs to be a combinatorial approach for RA synergistic treatment, while fundamental studies are needed to understand the synergistic treatment effect and explore the underlying mechanisms. To optimize IONPs as a RA therapeutic platform, better designed IONPs shall be constructed to improve the magnetic heating efficiency and enable the clinical application in MHT. The application of IONPs in RA therapy not only provides an effective approach for RA diagnosis and treatment but may also encourage the development of novel magnetic materials with higher performance potential.

Financial \& competing interests disclosure

This work was supported by the National Natural Science Foundation of China (81503017), Natural Science Foundation of Jiangsu Province (BK20150534). The authors have no other relevant affiliations or financial involvement with any organization or entity with a financial interest in or financial conflict with the subject matter or materials discussed in the manuscript apart from those disclosed.

No writing assistance was utilized in the production of this manuscript.

Author contributions

$\mathrm{L}$ Wu wrote the first five paragraphs. S Shen wrote the following parts of the article.

\section{References}

1. Firestein GS. Evolving concepts of rheumatoid arthritis. Nature 423(6937), 356-361 (2003).

2. Ochoa CD, Stevens T. Studies on the cell biology of interendothelial cell gaps. Am. J. Physiol. 302(3), L275-L286 (2011). 
3. Wang J, Chen Y, Chen B et al. Pharmacokinetic parameters and tissue distribution of magnetic $\mathrm{Fe}_{3} \mathrm{O}_{4}$ nanoparticles in mice. Int. J. Nanomed. 5, 861-866 (2010).

4. Prijic S, Sersa G. Magnetic nanoparticles as targeted delivery systems in oncology. Radiother. Oncol. 45(1), 1-16 (2011).

5. Zhang S, Wu L, Cao J et al. Effect of magnetic nanoparticles size on rheumatoid arthritis targeting and photothermal therapy. Colloids. Surf. B 170, 224-232 (2018).

6. He C, Hu Y, Yin L, Tang C, Yin C. Effects of particle size and surface charge on cellular uptake and biodistribution of polymeric nanoparticles. Biomaterials 31(13), 3657-3666 (2010).

7. Treuhaft PS, McCarty DJ. Synovial fluid pH, lactate, oxygen and carbon dioxide partial pressure in various joint diseases. Arthritis Rheum. 14(4), 475-484 (1971).

8. Simon GH, von Vopelius-Feldt J, Fu Y et al. Ultrasmall supraparamagnetic iron oxide-enhanced magnetic resonance imaging of antigen-induced arthritis: a comparative study between SHU 555 C, ferumoxtran-10, and ferumoxytol. Invest. Radiol. 41(1), 45-51 (2006).

9. Simon GH, von Vopelius-Feldt J, Wendland MF et al. MRI of arthritis: comparison of ultrasmall superparamagnetic iron oxide vs Gd-DTPA. Gd-DTPA J. Magn. Reson. Imaging 23(5), 720-727 (2006).

10. Wei H, Bruns OT, Kaul MG et al. Exceedingly small iron oxide nanoparticles as positive MRI contrast agents. Proc. Natl Acad. Sci. USA 114(9), 2325-2330 (2017).

11. Lee J-H, Jang J-t, Choi J-s et al. Exchange-coupled magnetic nanoparticles for efficient heat induction. Nat. Nanotech. 6(7), 418-422 (2011).

12. Shen $\mathrm{S}$, Kong F, Guo X et al. CMCTS stabilized $\mathrm{Fe}_{3} \mathrm{O}_{4}$ particles with extremely low toxicity as highly efficient near-infrared photothermal agents for in vivo tumor ablation. Nanoscale 5(17), 8056-8066 (2013).

13. Hitchon CA, El-Gabalawy HS. Oxidation in rheumatoid arthritis. Arthritis Res. Ther. 6(6), 265-278 (2004).

14. Thoidingjam S, Tiku AB. New developments in breast cancer therapy: role of iron oxide nanoparticles. Adv. Nat. Sci. 8(2), 023002 (2017).

15. Dabbagh AJ, Trenam CW, Morris CJ, Blake DR. Iron in joint inflammation. Ann. Rheum. Dis. 52(1), 67-73 (1993). 